\title{
HEAT TRANSFER ANALYSIS IN A ROTATING CAVITY WITH AXIAL THROUGH-FLOW
}

\author{
M.R. Puttock-Brown* \\ C.A. Long \\ Thermo-Fluid Mechanics Research Centre \\ School of Engineering and Informatics \\ University of Sussex \\ Falmer, BN1 9QT \\ United Kingdom \\ Email: M.Puttock-Brown@sussex.ac.uk
}

\section{ABSTRACT}

This paper presents local Nusselt numbers computed from experimental measurements of surface temperature of compressor discs in a multiple rotating cavity test rig with axial throughflow. A validated 2D steady state heat conduction analysis methodology is presented, using the actual test geometry, and 95\% confidence intervals calculated using Monte Carlo simulation. Sensitivity of the solution to curve fitting types, geometric simplification and surface instrumentation are explored.

The results indicate that polynomial curves fits, whilst computational simple, are unsuitable especially at higher orders. It is shown that geometric simplifications, that typically simplify the algorithmic implementation, may also omit significant variation in heat flux at critical stress relieving locations. The effect of reducing measurement points in the analysis is to both over-predict heat transfer and increase the uncertainty of the results. Finally, the methodology is applied to previously published thermal data from the University of Sussex, facilitating qualitative discussion on the influence of the governing parameters.

Whilst this study does not overcome the inherent uncertainty associated with inverse solutions it is intended to present a methodology that is readily available to the wider community for the analysis of thermal test data and suggests some guidelines at the planning and post-processing stages.
The range of experiment reported here covers: $1.13 \times 10^{5}<$ $\operatorname{Re}_{z}<5.14 \times 10^{5}, 1.65 \times 10^{6}<\operatorname{Re}_{\theta}<3.16 \times 10^{6}, 0.10<\operatorname{Ro}<$ 0.60 and $3.40 \times 10^{11}<\mathrm{Gr}<1.25 \times 10^{12}$.

\section{NOMENCLATURE}

$a \quad$ Disc inner radii $[\mathrm{m}]$

$b \quad$ Disc outer radii $[\mathrm{m}]$

$D \quad$ Rotor Outer Diameter [m]

Gr Grashof Number $\left(=\rho \Omega^{2} b \beta \Delta T L^{3} / \mu^{2}\right)$

$k \quad$ Thermal conductivity [W $\mathrm{W}^{-2} \mathrm{~K}$ ]

$q \quad$ Heat Flux $\left[\mathrm{W} \mathrm{m}^{-2}\right.$ ]

$\operatorname{Re}_{z} \quad$ Axial Reynolds Number $\left(=\rho W d_{h} / \mu\right)$

$\operatorname{Re}_{\theta} \quad$ Reynolds Number $\left(=\rho \Omega b^{2} / \mu\right)$

Ro Rossby Number $(=W / \Omega a)$

$T \quad$ Static temperature [K]

$r \quad$ Radius [m]

$\beta \quad$ Coefficient of Volume Expansion $(=1 / T)\left[\mathrm{K}^{-1}\right]$

$\mu \quad$ Viscosity $\left[\mathrm{kg} \mathrm{m}^{-1} \mathrm{~s}^{-1}\right]$

$\rho \quad$ Density $\left[\mathrm{kg} \mathrm{m}^{-3}\right]$

$\Omega \quad$ Rotational speed $\left[\mathrm{rad} \mathrm{s}^{-1}\right]$

\section{Abbreviations}

MCR Multiple Cavity Rig

PDE Partial Differential Equation 


\section{INTRODUCTION}

The calculation of heat transfer coefficients from surface temperature measurements is a perennial problem. Small errors in the recorded temperature lead to large errors in the calculated heat transfer as a consequence of computing temperature gradients within the body. This inverse problem is well documented in literature. Despite this, it is common practise to use experimental measurements in calculating heat fluxes. Such analysis typically rely on finite difference approximations in term-by-term replacement of the governing equation to determine the interior temperature distribution. This in turn is applied to simplified geometries, usually rectangular, that approximate the true geometry under study as this allows structured grids and subsequently reduced coding.

Previously, Owen [1] used fitted curves to the data points to smooth the data and minimise uncertainty in calculated heat fluxes whilst Cooke et al. [2] extended this using Monte Carlo simulation. Both used finite-difference approximations applied to simplified grids representative of turbine/compressor discs of gas turbine engines, similiar approaches were followed by Alexiou [3] and Patounas [4]. In each case the geometry was simplified, though this was extended to consider a variable crosssectional area using ANSYS in [4]. Across these, multiple curve fitting types are considered; cubic spline and n-order polynomials and are used to interpolate temperature measurements to arbitrary nodal locations with varying degrees of success. The number of data points used necessarily limits the order of the polynomial fit, historically this limit is the number of measurement points that can be physically instrumented on a rotating surface.

Recently Tang et al. [5] considered the difficulty of applying the inverse problem in such cases using Bayesian methods. They reported smoothly varying Nusselt number distributions using temperature data from Atkins and Kanjirakkad [6] alongside $95 \%$ confidence intervals. This approach uses the fin equation to provide information at the Biot number from which Nusselt numbers were obtained. For a general review of buoyancy-induced flow in rotating cavities the reader is directed to Owen and Long [7]. For detailed analysis of heat transfer in rotating cavity systems with axial throughflow the reader is directed, among others, to [8], [9] and [10]

This paper sets to establish a framework suitable for the analysis of data from experimental rigs focused towards rotating disc systems representative of gas turbine engine. It combines the use of realistic, and not simplified, geometries alongside demonstrating the advantages of increased instrumentation density and alternate curve fitting strategies and Monte Carlo simulation. All work presented in this paper uses the MATLAB ${ }^{\circledR}$ Partial Differential Equation Toolbox ${ }^{\mathrm{TM}}$, this is a collection of functions for the solution of PDEs using finite element analysis [11]. This is applicable to arbitrary $2 \mathrm{D}$ and $3 \mathrm{D}$ geometries that would be considerably more difficult to model using finite-difference approximations.

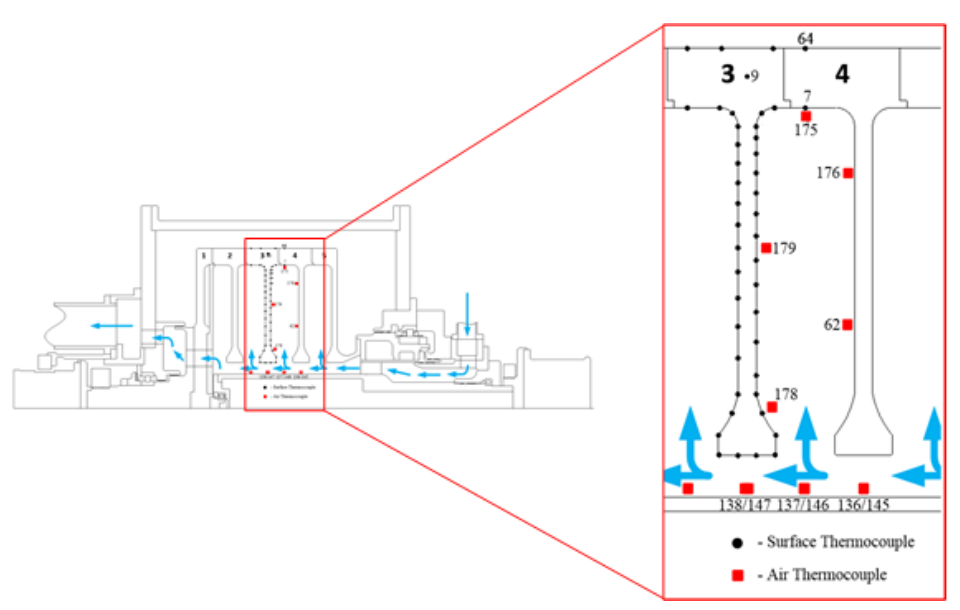

FIGURE 1. 2D CROSS SECTION OF THE MCR FACILITY

\section{EXPERIMENTAL FACILITY}

The thermal boundary conditions used in this paper were obtained using the Multiple Cavity Rig (MCR) at the University of Sussex. Full rig details are reported in [12], with essential information repeated here. Figure 1 shows a 2D cross-section schematic of the primary instrumented MCR cavity, with the insert showing thermal instrumentation. Embedded metal thermocouples are indicated by black circles and air thermocouples as red squares. In all cases the thermocouples are glass-fiber insulated K-type with a nominal bead diameter of $0.4 \mathrm{~mm}$, measured via radio telemetry with a total system uncertainty of $\pm 0.5 \mathrm{~K}$, as detailed in [6].

\section{METHODOLOGY OF DATA PROCESSING EQUATION AND SOLVER}

In an axisymmetric domain, such as a rotating disc it is convenient to use Laplace's equation in cylindrical coordinates:

$$
\frac{1}{r} \frac{\partial}{\partial r}\left(k r \frac{\partial T}{\partial r}\right)+\frac{1}{r^{2}} \frac{\partial}{\partial \theta}\left(k \frac{\partial T}{\partial \theta}\right)+\frac{\partial}{\partial z}\left(k \frac{\partial T}{\partial z}\right)=0
$$

where $r$ is the radius, $\theta$ the circumferential and $z$ the axial coordinate. Assuming the system is thermally axisymmetric, then $\partial T / \partial \theta=0$, which reduces Equation 1 to;

$$
\frac{1}{r} \frac{\partial}{\partial r}\left(k r \frac{\partial T}{\partial r}\right)+\frac{\partial}{\partial z}\left(k \frac{\partial T}{\partial z}\right)=0
$$

Finally, the cylindrical form of Equation 2 required by the PDE Toolbox (note the additional $r$ term) is given as;

$$
\nabla \cdot(k r \nabla T)=0
$$


The surface normal heat flux is calculated from both radial and axial components. The total heat flux $q$ given is the summation of both the conductive and radiative, such that:

$$
q=q_{c}+q_{r}
$$

The radiative component $q_{r}$ is calculated using view factors [13] and the method detailed in [14]. This is subtracted from the total to give the conductive heat flux required for the calculation of Nusselt number, defined as:

$$
\mathrm{Nu}=\frac{q_{c} L}{k\left(T-T_{r e f}\right)}
$$

where $q_{c}$ is the local wall normal conductive heat flux, $T$ the local wall temperature, $k$ the thermal conductivity and $L$ a relevant length scale. Typically the length scale when evaluating the local Nusselt numbers for rotating discs is the radius $r$. For rotating cavity systems, the axial throughflow temperature is commonly used as the reference air temperature $T_{r e f}$.

Unless specified, all data presented in this section uses Case A $\left(\mathrm{Gr}=3.58 \times 10^{11}\right.$, Ro $\left.=0.59\right)$ as a reference case. Results pertaining to the wider test programme are presented later.

\section{GEOMETRY AND BOUNDARY CONDITIONS}

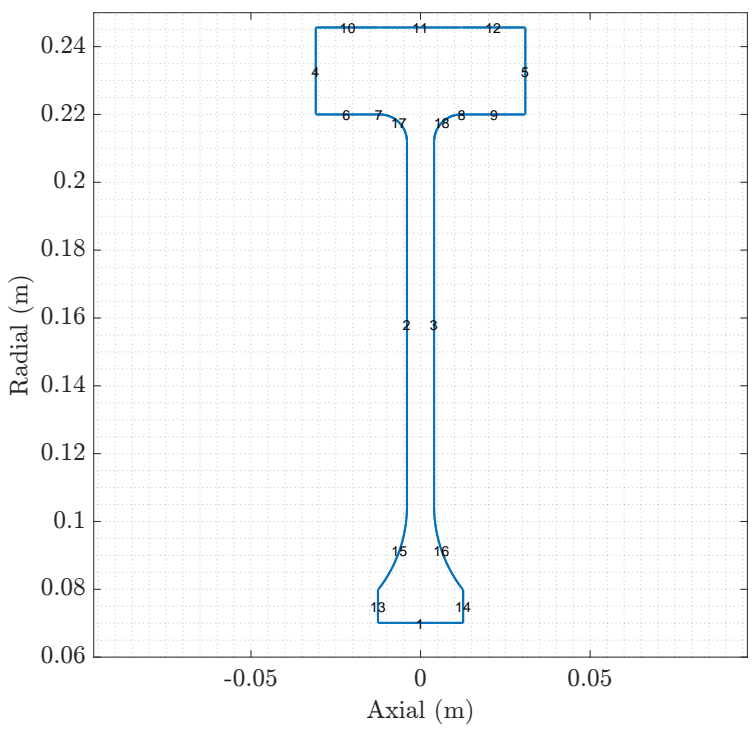

FIGURE 2. DOMAIN GEOMETRY - The edge numbers indicate the boundary condition labels
Heat conduction modelling is performed using the geometry of the MCR discs, this allows geometric components such as cob, shroud and rim alongside the diaphragm to be modelled, as shown in Figure 2. This is an accurate geometric representation of the MCR disc, and contrary to previous work that assumed a constant thickness disc [15].

Isothermal experimental temperatures are used on all domain boundaries, including the outer rim. Boundaries 4 and 5 in Figure 2 (between the outer rim and shroud) are adiabatic, on the assumption the heat flux is predominately radial. Thermal conductivity is constant at $7.71 \mathrm{~W} / \mathrm{mK}$.

\section{VALIDATION}

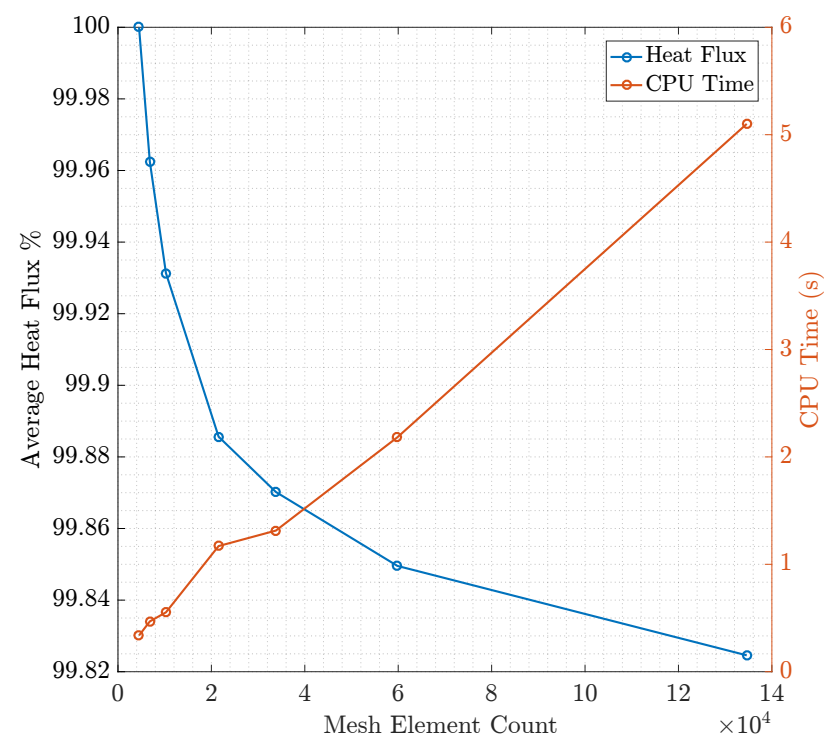

FIGURE 3. GRID INDEPENDENCE STUDY - Based on surface average heat flux, secondary axis indicates solution time

Figure 3 show the grid independence study conducted using a second order exponential fit to experimental temperatures. This is defined as:

$$
T=a e^{b r}+c e^{d r}
$$

where $a, b, c, d$ are constants used in the fit. There is no significant difference in average surface heat flux with increasing mesh size relative to the coarsest mesh. There is however a significant linear trend in CPU run time that must be considered in light of conducting a Monte Carlo analysis. For this reason, the 35,000 element mesh is used, corresponding to a maximum 


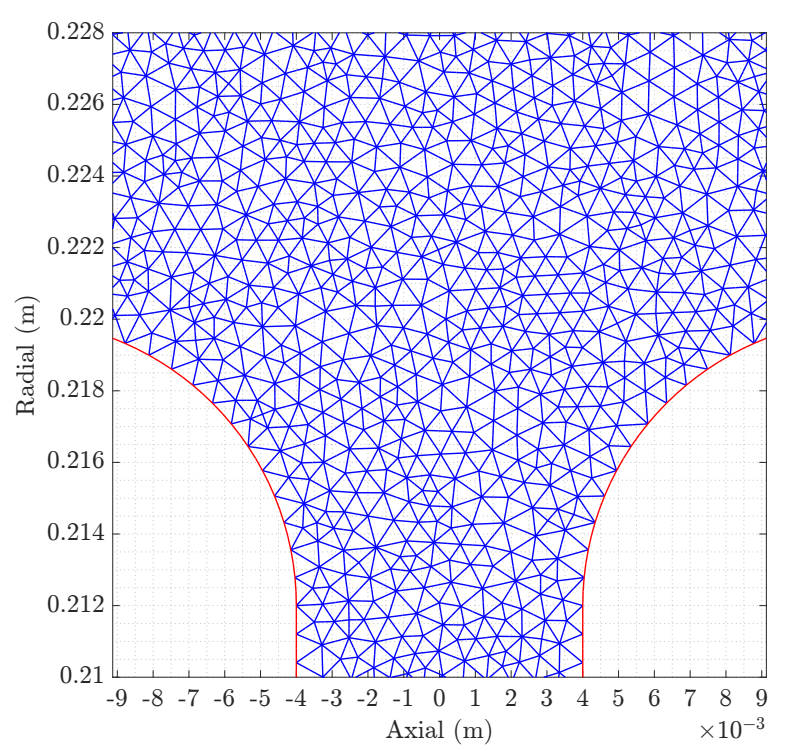

FIGURE 4. FINITE ELEMENT MESH - Maximum edge length = $0.8 \mathrm{~mm}$, area shown is upper fillet radii

element edge length of $0.8 \mathrm{~mm}$. The advantage of an unstructured solver is illustrated in the ability to closely approximate the curved parts of the domain (Figure 4).

Validation is considered firstly via comparison to an internal thermocouple (\# 9) located between the rim and shroud at the disc midpoint (Figure 1). For all tests considered in the programme, the numerical results agree to within $2 \mathrm{~K}$ (Figure 5a), this is considered sufficient in light of both the experimental uncertainty and the inability of the solver to account for some the physical non-axisymmetric features of the rig including instrumentation passages and disc-pack boltholes.

The second validation, after Long and Childs [16], considers the 1D axial heat flux using opposed thermocouples on the shroud and outer surface (indicated as \#7 and \#64 in Figure 1) compared to

$$
q_{s h}=\frac{k\left(T_{64}-T_{7}\right)}{b \ln (D / 2 b)}
$$

Figure 5 compare the computed and calculated shroud heat fluxes. The mean agreement for all tests is within $8 \%$, this improves to within 3\% when considering the Phase B testing which was conducted after a rebuild and instrumentation of the rig (note, the instrumentation locations were unchanged). Again, considering the non-axisymmetric features not modelled and that Equation 7 ignores any axial heat flux this is considered sufficient to give confidence in the result. The increase in the agreement between the phases is likely due to the increased number of
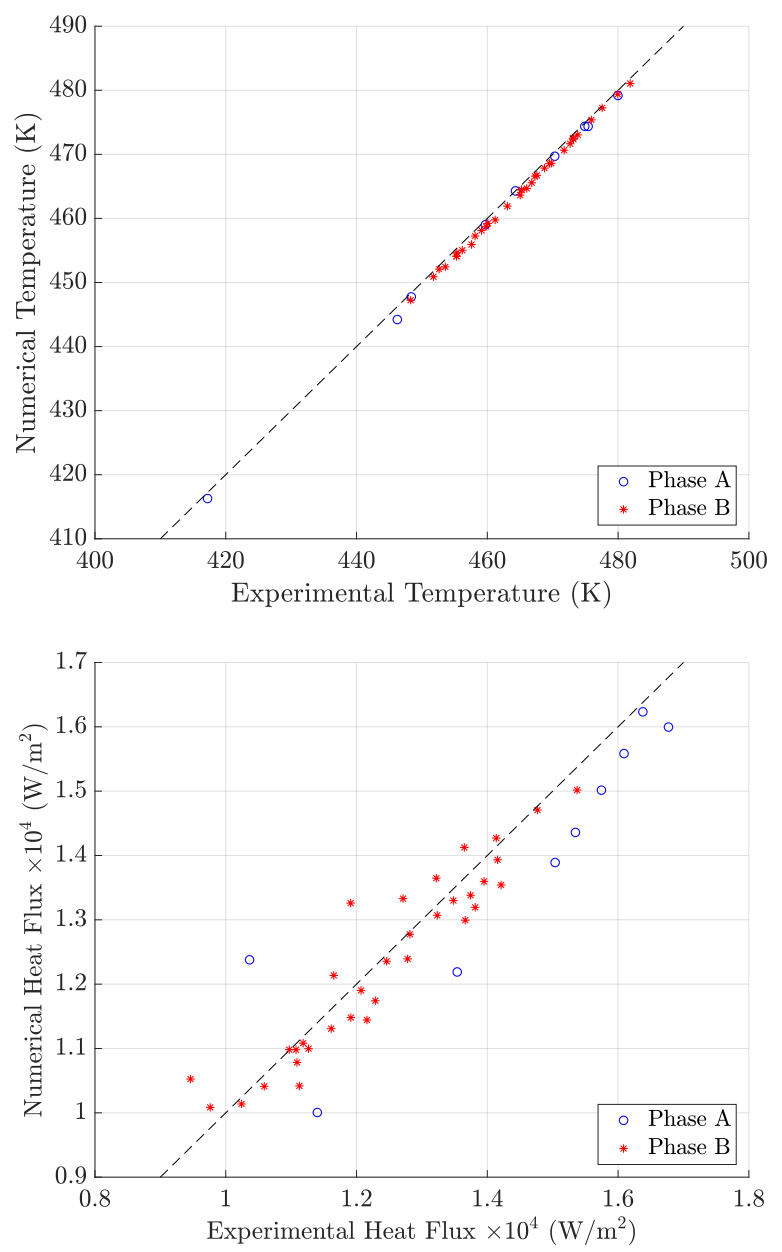

FIGURE 5. VALIDATION COMPARISON - a) Comparison to thermocouple \#9, b) - Shroud heat flux comparison

functioning thermocouples on the disc surface.

\section{MONTE CARLO ANALYSIS}

It is well known that deriving heat transfer coefficients, or Nusselt numbers, from experimentally measured surface temperatures is extremely sensitive to small perturbations, this is the inverse problem. Traditionally, this is exacerbated by the lack of surface measurements on rotating components as small perturbations in the curve fit will result in large uncertainty in the Nusselt numbers. With the number of measurements present in the current programme it becomes significantly more difficult to evaluate Nusselt number uncertainty based on individual experimental measurement uncertainty.

Monte Carlo simulation has been used extensively, initially in radiative heat transfer (Howell [17]) and in inverse heat conduction problems using random walk (Haji-Sheikh and Bucking- 
ham [18]). In the context of this paper, Monte Carlo simulation is used as a stochastic alternative to Taylor series uncertainty propagation analysis, which requires independent parameters and is considerably more complicated. It has been previously shown to offer practical results for evaluating disc surface heat fluxes [2]. To conduct a Monte Carlo simulation the model is repeatedly run, on the same mesh, using boundary conditions based on experimentally measured temperatures modified with a randomly generated (Gaussian probability distribution) uncertainty within the bounds of $\pm 0.5 \mathrm{~K}$. For each test case, the solver is run 10,000 times, which is considered sufficient [19] and the surface normal heat fluxes recorded. A second simulation is used to assess the surface Nusselt numbers, by applying a random uncertainty to the upstream reference air temperature (\# 136, Figure 1) to give both local and average disc Nusselt numbers.

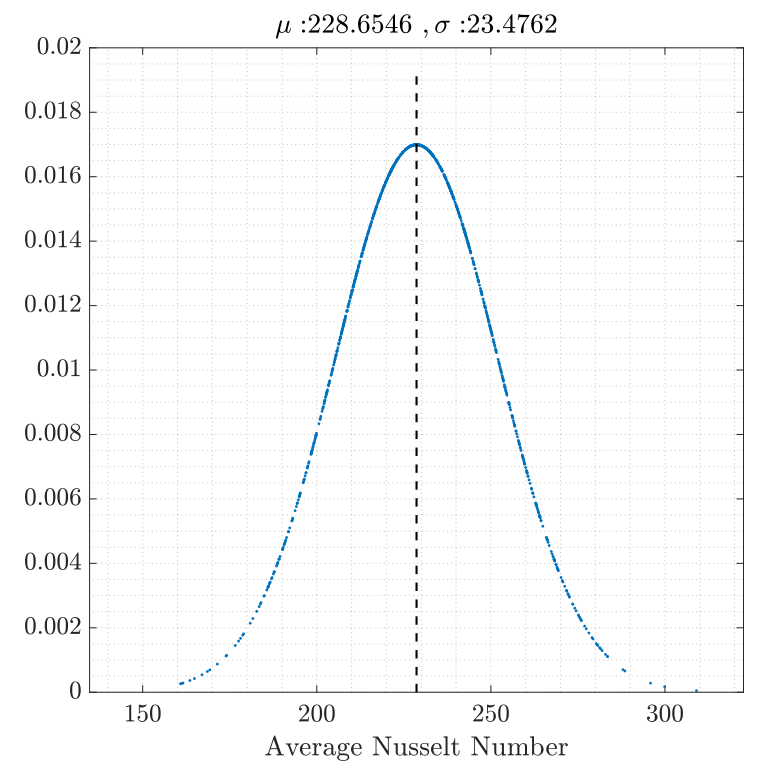

FIGURE 6. RADIALLY WEIGHTED AVERAGE DISC NUSSELT NUMBER - Calculated using Monte Carlo simulation

Figure 6 shows the radially weighted average Nusselt number of Case A using Monte Carlo simulation. By fitting a suitable probability distribution, the mean and standard deviation can be calculated and used to report the true mean alongside the $95 \%$ confidence interval (1.96 standard deviations). In deriving the local Nusselt numbers, this procedure can be repeated at each evaluation point to give both the mean and $95 \%$ confidence interval over the entire surface of the disc. Figure 7 shows this applied to test case A, the relevant physics will be discussed later.

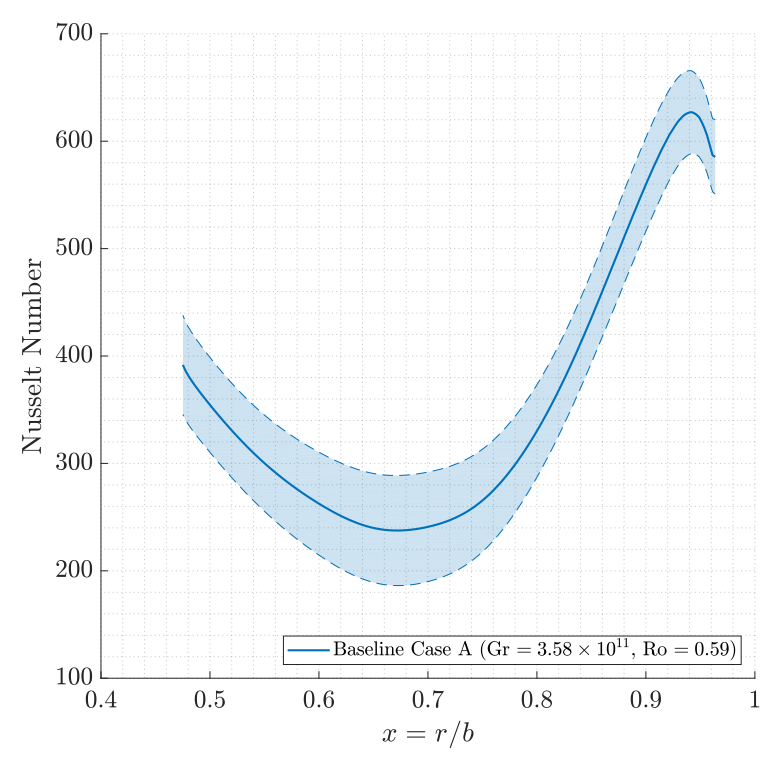

FIGURE 7. LOCAL NUSSELT NUMBERS - Solid line indicates mean value. Shaded area indicates $95 \%$ confidence interval.

\section{SENSITIVITY ANALYSIS}

With an established procedure for the processing of thermal data via Monte Carlo simulation, the sensitivity to various parameters can be assessed. This section will look at three sensitivities; type of curve fit to experimental measurements, geometry and instrumentation density.

CURVE FIT Previously a number of different curve types have been fitted to temperature measurements on a rotating disc, this paper will only consider three types: a 5th order polynomial, a cubic spline and a second-order exponential curve. Despite all showing low RMS error in temperature (of order $<0.02$ $\mathrm{K}$ [12]) the Nusselt number profiles show significant variation. In all cases they are compared over the disc diaphragm section for comparison to data sets in literature, in the current setup this corresponds to $0.47<x<0.97$.

Figure 8 shows the three curve fit types for Case A. It is apparent that the 5th-order polynomial is erroneous; there are multiple turning points (likely due to the oscillations in the polynomial) and a large confidence interval. Whilst the spline fit shows a smooth variation decreasing towards the bore region, the sharp and significant drop at high radius is contrary to current understanding. The profile of the exponential fit shows a tighter confidence interval than either polynomial or spline fits with a smooth radial variation. The pronounced upward trend from approximately $x=0.7$ is similar to reported trends in [10] who notably also used a 2D solution.

Figure 9 shows the comparison of the different fit types to 


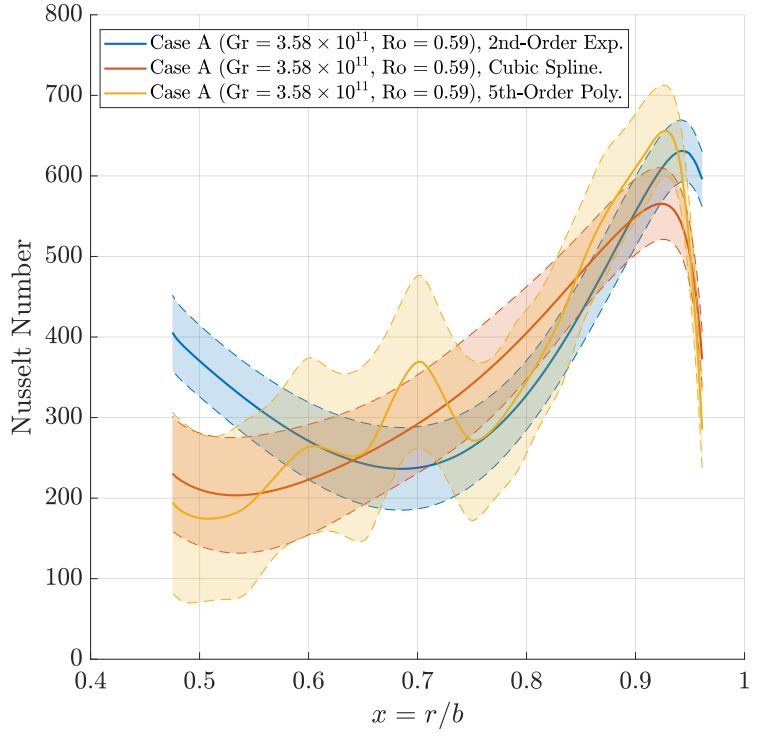

FIGURE 8. NUSSELT NUMBER CURVE FIT COMPARISON - Fit types considered: second-order exponential, 5th-order polynomial and cubic spline

the prediction of temperature at the validation point (measurement \# 9), whilst this point is outside of the diaphragm there is an impact of the fit type. In all cases considered in the test programme the exponential fit shows the lowest mean variation from the experimental values.

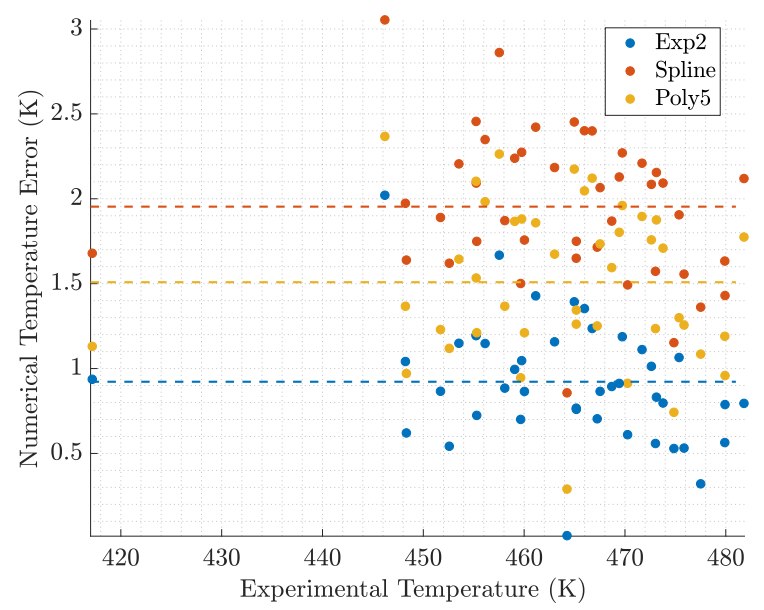

FIGURE 9. CURVE FITTING ABSOLUTE ERROR - Comparison of Curve fit types to experimental values of thermocouple \#9
GEOMETRY COMPARISON In previous work on rotating discs the geometry is either rectangular or simplified, modelled as a 2D cross-section of constant thickness. This approach lends itself well to the finite-difference method on a regular grid however; this does not take into account important stress relieving features such as the cob and fillet radii at high and low radius. To consider this, the solver is used to investigate the sensitivity of the Nusselt numbers to three variants of the geometry; full, reduced and clipped with the exponential type fit. The full variant (shown in Figure 2) is the most representative, the reduced uses a constant cross-section area along the entire disc but retains the rim and the clipped considers just the disc with constant cross sectional area. Considered geometries are shown in Figure 10.

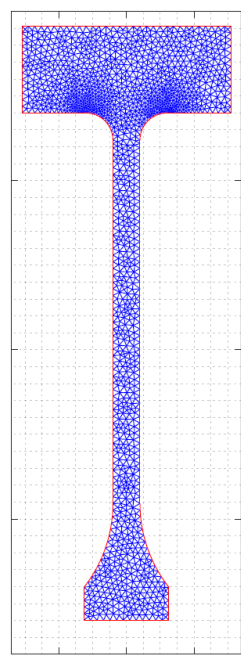

Full

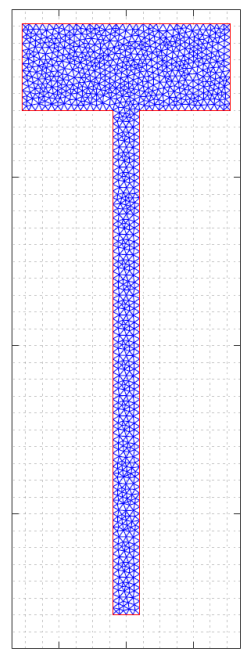

Reduced

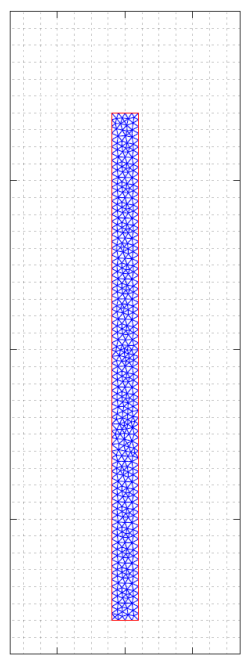

Clipped
FIGURE 10. GEOMETRY COMPARISON

Figure 11 shows the Nusselt number comparison from all geometries, to appreciate the differences the entire disc length is shown. Significantly along the disc diaphragm all three geometries agree and indicate similar confidence intervals. The effect of the shroud corner (the upper fillet radii joining the diaphragm to the shroud) is clear, with a substantial peak in Nusselt number with a tight confidence interval. The other two geometries quickly tend to a decreasing Nusselt number (and increasing uncertainty), indicating a strong heat flux gradient in the area. The clipped geometry indicates negative numbers, which are considered erroneous. In the near bore region, the full geometry shows significant variation from the other two, this is due to the inclusion of the cob and fillet radii, which increases the cross sectional area and spreads the radial heat flux. The cob sides show an erroneous profile, indicated by the trend and large confidence interval. The small $\Delta T$ and Nusselt number sensitivity to temperature uncertainty mean the prediction in this area must be rejected. 


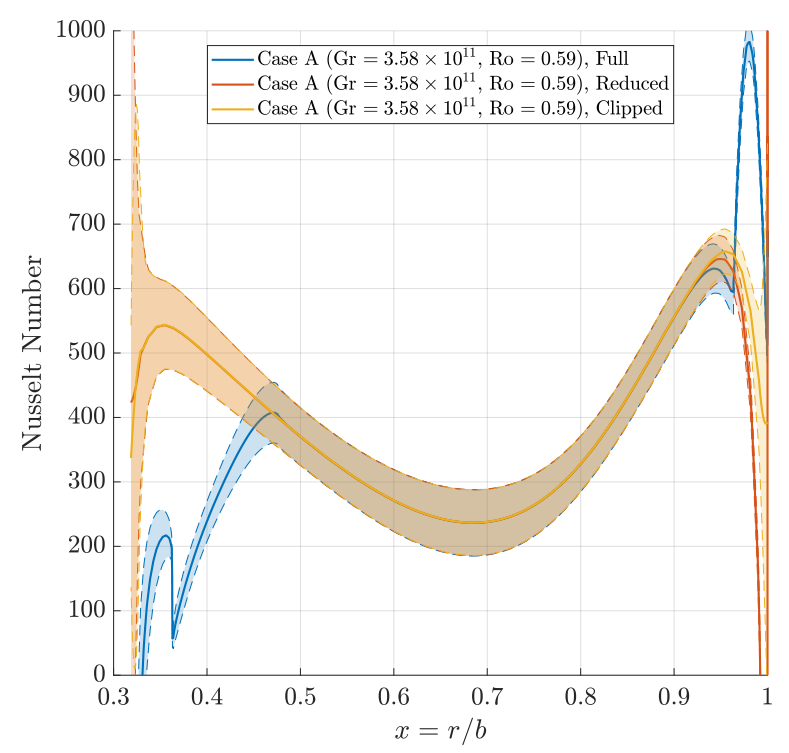

FIGURE 11. NUSSELT NUMBER GEOMETRY COMPARISON

THERMOCOUPLE DENSITY The final sensitivity study concerns the density of instrumentation points used in fitting the temperature profile. By reducing the number of measurement points used to fit the curve, the impact this has on both the Nusselt number prediction and confidence interval can be investigated. Monte Carlo simulation was again applied to the disc profile in Figure 2 using a second-order exponential fit but with a reduced number of measurement points. This was achieved by using every second and then every fourth measurement point. The first and last measurement point in the radial direction is retained to fix the upper and lower boundaries.

The Nusselt number profile in Figure 12 clearly illustrates that by decreasing the number of measurement points used in the temperature fit there is both an increase in Nusselt number in the outer part of the disc and that the confidence interval increases considerably. This result is unsurprising given that by using a reduced number of measurement point in the curve fit, the fit becomes less restrained leading to a larger spread in the results and is consistent with [2].

\section{RESULTS \& DISCUSSION}

The methodology outline in the previous section is applied to results previously published in [20]. Results of Phase B of testing in the programme are not included for reasons of commercial sensitivity. The results cover the test matrix presented in Table 1 and will focus on the trends of the Nusselt number profiles rather than the temperature distributions as these have been reported previously.

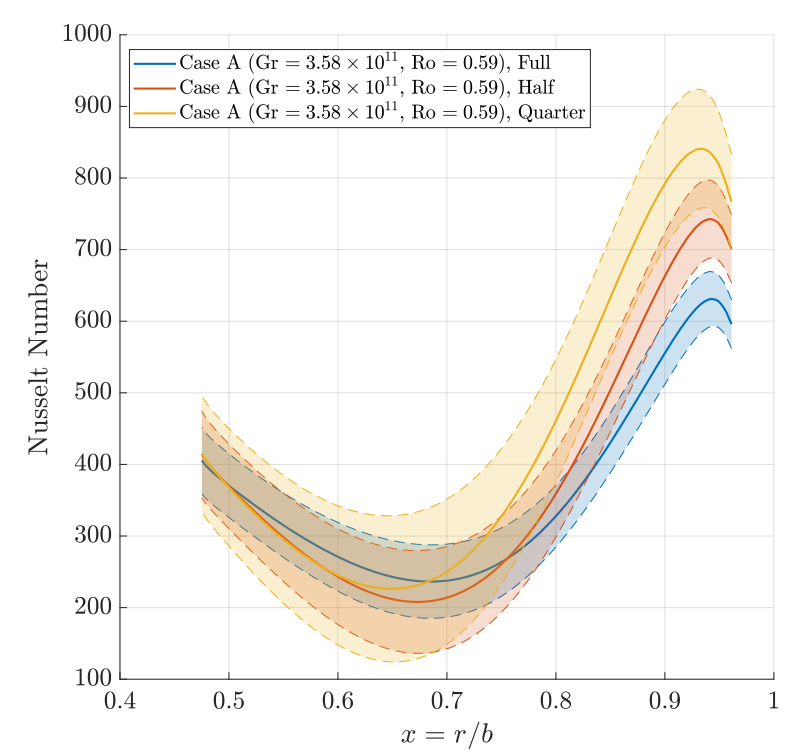

FIGURE 12. NUSSELT NUMBER THERMOCOUPLE COMPARISON - Comparing results of different thermocouple densities used in curve fit

\begin{tabular}{lc}
\hline \hline Axial Reynolds Number & $1.13 \times 10^{5}<\mathrm{Re}_{z}<5.14 \times 10^{5}$ \\
Rotational Reynolds Number & $1.65 \times 10^{6}<\mathrm{Re}_{\theta}<3.16 \times 10^{6}$ \\
Rossby Number & $0.10<\mathrm{Ro}<0.60$ \\
Buoyancy Parameter (shroud) & $0.32<\beta \Delta T<0.40$ \\
Buoyancy Parameter (diaphragm) & $0.13<\beta \Delta T_{a v}<0.17$ \\
Grashof Number & $3.40 \times 10^{11}<\mathrm{Gr}<1.25 \times 10^{12}$ \\
\hline
\end{tabular}

TABLE 1. Range of nondimensional parameters for Phase A

Figures 13 - 15 show the nondimensional temperatures and upstream disc surface local Nusselt numbers, in order of decreasing Rossby number. The nondimensional temperature is defined as:

$$
\Theta=\frac{T_{s}-T_{i n}}{T_{s h}-T_{i n}}
$$

where $T_{\text {in }}$ uses thermocouple \#136 upstream of the cavity inlet, $T_{s h}$ is \#7 and $T_{s}$ the local disc surface temperature (Figure 1). In some of the Figures $\Theta>1$, this is due to the use of shroud temperature in the upstream cavity. This explains why $\Theta>1$ is only indicated on the downstream temperature profiles. The local 

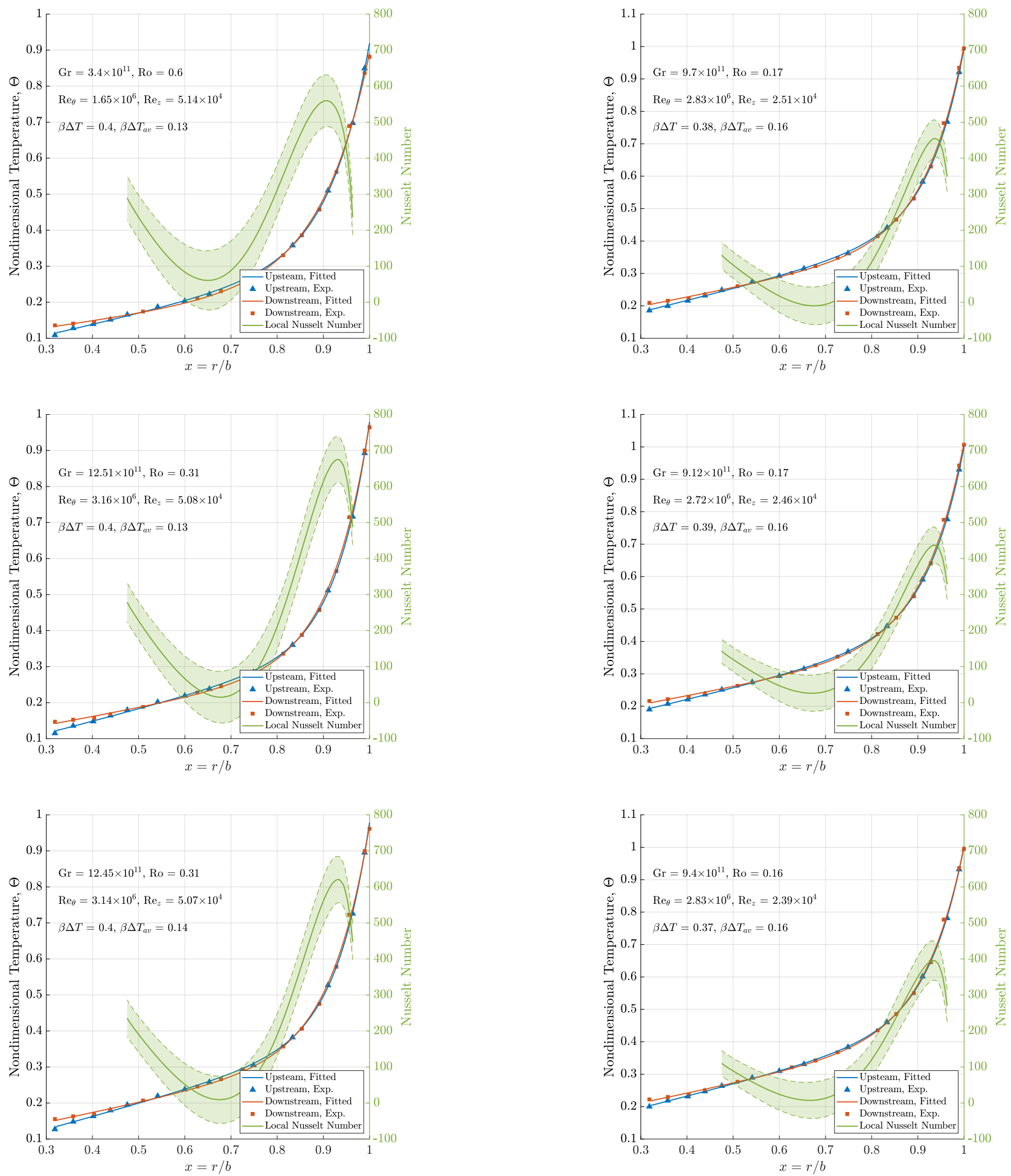

FIGURE 13. LOCAL NUSSELT NUMBER and $\Theta$ DISTRIBUTIONS - Phase A, $0.31 \leq$ Ro $\leq 0.60$

FIGURE 14. LOCAL NUSSELT NUMBER and $\Theta$ DISTRIBUTIONS - Phase A, $0.16 \leq$ Ro $\leq 0.17$ 

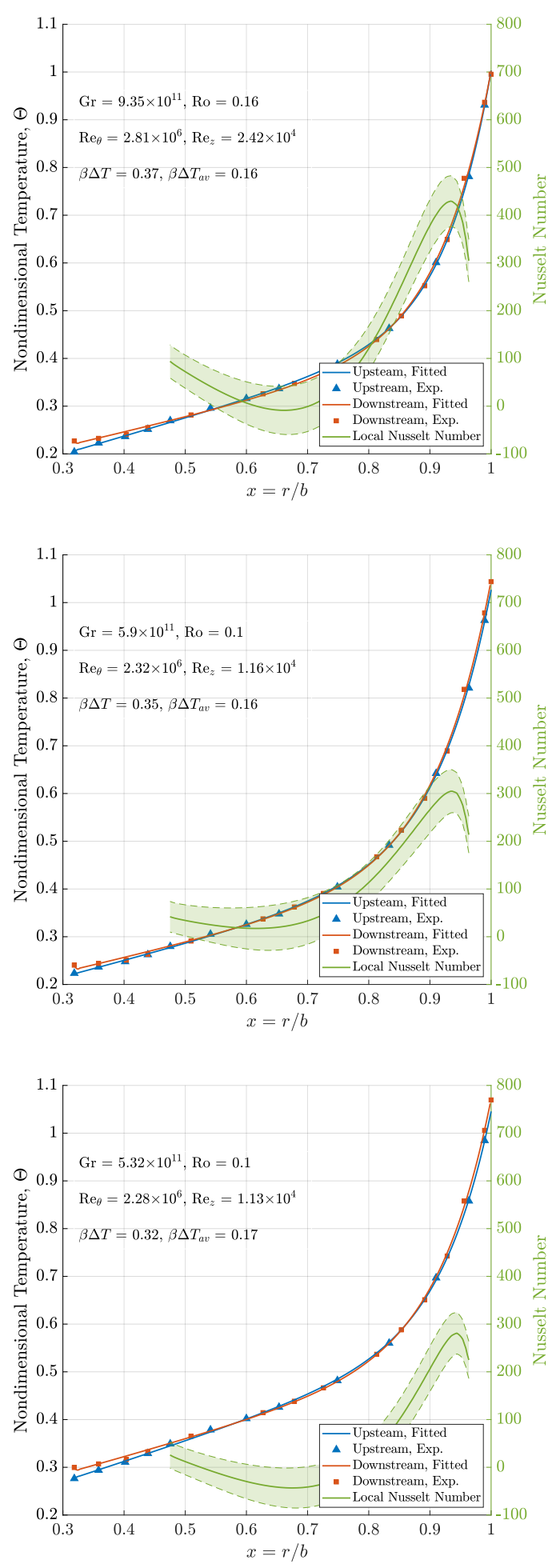

FIGURE 15. LOCAL NUSSELT NUMBER and $\Theta$ DISTRIBUTIONS - Phase A, $0.10 \leq$ Ro $\leq 0.16$
Nusselt number is defined as:

$$
\mathrm{Nu}=\frac{q_{c} r}{k_{\text {air }}\left(T_{s}-T_{\text {in }}\right)}
$$

where $T_{\text {in }}$ uses thermocouple \#136 and $q_{c}$ has been corrected for radiation. In all cases Monte Carlo simulation has been conducted (with $n=10000$ ) given 95\% confidence intervals, shown as shaded areas.

The distinctive shape of the Nusselt number distribution is seen across all tests, in all cases there is a local minima near $x=0.65$ that increases towards the bore and shroud. Similar results were found in [10] who noted a minima in heat flux in the same $x$ region. As the Rossby number decreases the depth of this minima decreases as the Nusselt number range diminishes. This is consistent with the reduction of the overall magnitude of Nusselt number, indicating lower levels of heat transfer. With relatively similar levels of $\beta \Delta T$ across the tests, this suggests that as the flow structure becomes more rotationally dominated (as Rossby reduces) the buoyancy driven convection becomes stronger relative to forced convection. This in turn promotes mixing in the cavity, leading to a more homogeneous radial temperature distribution, suppressing heat transfer; indeed the disc temperatures at lower Rossby are more uniform.

It is also significant that the minima corresponds with zero or negative Nusselt numbers, this is due to the overlap of the temperature distributions across the disc walls. In this region, in all considered cases, the temperature on the upstream face is hotter than the downstream, resulting in a negative heat flux in the conduction model. Given the relatively close spacing of the measurements and the applied uncertainty, this region is sensitive to the curve fit and so the largest confidence intervals are in this region.

At the lower disc radii, the Nusselt number tends to increase; this is consistent with an impingement of the cooler forced convection throughflow onto the disc surface.

\section{CONCLUSIONS}

This paper has applied 2D finite element analysis to the solution of internal steady state heat conduction. Monte Carlo simulation was conducted using experimentally obtained data points (temperature measurements) radially spaced on a rotating disc representative of gas turbine engine high-pressure compressor disc. With an established method, sensitivity tests were conducted to assess the impact of geometry, curve fitting type and number of data points on the magnitude of the uncertainty and shape of the trend.

Regarding the type of fit used, the second-order exponential shows improved results when compared to both spline and polynomial fit types. This is based on displaying similar magnitudes of heat transfer with reduced uncertainty and consistency with 
previous work. When looking at geometric variation the results indicate agreement in the diaphragm region (where the geometries are identical), however when considering the design of gas turbines important, stress relieving, features should be considered. The actual geometry shows considerable variation in the upper fillet radii, indicating a peak in heat transfer magnitude with a tighter confidence interval. It is suggested that instrumentation in this feature is considered in detail when planning future rotating cavity experiments.

Unsurprisingly, decreasing the number of data points in the curve fitting increases the uncertainty as this allows more variance in the fit. The peak magnitude in Nusselt number also increases by approximately $30 \%$ when reducing the number of data points used to a quarter of their initial value.

The essential difficulty of inverse solution to obtain heat fluxes from surface temperature measurements is not mitigated. There is still considerable uncertainty in the reported Nusselt numbers; however, the reported methodology does facilitate qualitative investigation of the Nusselt number profile, especially when considered across a range of key non-dimensional parameters. It is recommended that with inverse analysis the full geometry is considered and that polynomial curve fitting be avoided (or at least use a lower order as possibly to prevent oscillation). Monte Carlo simulation proves an effective tool to establish confidence and, given modern desktop computing power, is easily achievable. Also, by demonstrating a workflow entirely within the MATLAB ${ }^{\circledR}$ software package it is readily available to the industry and academic communities. Of final note, the authors acknowledge that a more representative estimation of the heat transfer coefficient may be obtained by using all air thermocouples shown in Figure 1 and, although not used here, may be considered in future work.

\section{REFERENCES}

[1] Owen, J. M., 1979. "On the computation of heat-transfer coefficients from imperfect temperature measurements". Journal Mechanical Engineering Science, 21(5), pp. 323334.

[2] Cooke, A., Childs, P., and Long, C., 2006. “An investigation into the uncertainity of turbomachinery disc heat transfer calculations using monte carlo simulation". ASME Paper No. GT2006-90143.

[3] Alexiou, A., Hills, N. J., Long, C., Turner, A. B., and Millward, J. A., 2000. "Heat transfer in high-pressure compressor gas turbine internal air systems: A rotating disc-cone cavity with axial throughflow". Experimental Heat Transfer, 13(4), pp. 299-328.

[4] Patounas, D. S., Long, C. A., and Childs, P. R. N., 2009. "Disc heat transfer in gas turbine compressor internal air systems". In 8th European Conference on Turbomachin- ery: Fluid Dynamics and Thermodynamics, ETC 2009 Conference Proceedings, pp. 377-386.

[5] Tang, H., Shardlow, T., and Michael Owen, J., 2015. "Use of Fin Equation to Calculate Nusselt Numbers for Rotating Disks". Journal of Turbomachinery, 137(12), 09. 121003.

[6] Atkins, N. R., and Kanjirakkad, V., 2014. "Flow in a rotating cavity with axial throughflow at engine representative conditions". ASME Paper No. GT2014-27174.

[7] Owen, J. M., and Long, C., 2015. "Review of buoyancyinduced flow in rotating cavities". ASME. J. Turbomach., 137(11), p. 111001.

[8] Farthing, P. R., Long, C. A., Owen, J. M., and Pincombe, J. R., 1992. "Rotating cavity with axial throughflow of cooling air: Heat transfer”. ASME. J. Turbomach., 114(1), pp. 229-236.

[9] Owen, J. M., and Powell, J., 2006. "Buoyancy-induced flow in a heated rotating cavity". Journal of Engineering for Gas Turbines and Power, 128(1), pp. 128-134.

[10] Günther, A., Uffrecht, W., and Odenbach, S., 2012. "Local measurements of disk heat transfer in heated rotating cavities for several flow regimes". ASME J. Turbomach, 134(5), p. 051016.

[11] MATLAB, 2019. version 9.6.0 (R2019a). The MathWorks Inc.

[12] Puttock-Brown, M. R., 2018. "Experimental and numerical investigation of flow structure and heat transfer in gas turbine hp compressor secondary air systems". PhD Thesis, University of Sussex, Falmer.

[13] Howell, J. R., 1982. A Catalog of Radiation Configuration Factors. McGraw-Hill Book Company, New York.

[14] Long, C. A., 1999. Essential Heat Transfer. Longman.

[15] Alexiou, A., 2000. "Disc heat transfer in gas turbine h. p. compressors internal air systems". PhD Thesis, University of Sussex, Falmer.

[16] Long, C. A., and Childs, P. R. N., 2007. "Shroud heat transfer measurements inside a heated multiple rotating cavity with axial throughflow". Int. J. Heat Fluid Flow, 28(6), pp. 1405-1417.

[17] Howell, J. R., 1969. Application of Monte Carlo to Heat Transfer Problems, Vol. 5 of Advances in Heat Transfer.

[18] Haji-Sheikh, A., and Buckingham, F. P., 1993. "Multidimensional Inverse Heat Conduction Using the Monte Carlo Method". Journal of Heat Transfer, 115(1), 02, pp. 26-33.

[19] Coleman, H. W., and Steele, W. G., 1999. Experimental and Uncertainty Analysis for Engineers. John Wiley \& Sons.

[20] Tang, H., Puttock-Brown, M. R., and Owen, J. M., 2017. "Buoyancy-induced flow and heat transfer in compressor rotors”. ASME. J. Gas Turbines and Power. 